\title{
Neuer TKI zeigt Wirksamkeit bei EGFR-Resistenzmutation
}

\author{
Bei vielen Patienten mit nichtkleinzelligem Bronchialkarzinom \\ (NSCLC), die initial auf Tyrosinkinaseinhibitoren (TKI) \\ ansprechen, kommt es 1-2 Jahre nach Therapiebeginn zum \\ Progress. Dieser erworbenen Resistenz liegt in ca. $60 \%$ der \\ Fälle eine zusätzliche EGFR-T790M-Mutation zugrunde. \\ Die bisherigen Behandlungsoptionen sind durch geringe \\ Ansprechraten und dosislimitierende toxische Wirkungen \\ begrenzt. \\ N Engl J Med 2015; 372: 1689-1699
}

Die Daten der laufenden Studie von P.Jänne et al. haben nun die Wirksamkeit und Sicherheit eines neuen irreversiblen TKI (AZD9291) belegt, der neben aktivierenden EGFR-Mutationen auch auf die Resistenzmutation T790M abzielt. In die internationale Studie wurden Patienten mit lokal fortgeschrittenem oder metastasiertem NSCLC aufgenommen, wenn eine aktivierende EGFR-Mutation vorlag und die Erkrankung während einer EGFR-TKI-Behandlung fortschritt. Zunächst wurde ein Teil der Patienten auf Dosiseskalations-Kohorten randomisiert, in denen sie entweder 20, 40, 80, 160 oder 240 mg pro Tag AZD9291 erhielten. Diese Dosierungen wurden nach einer pharmakokinetischen Evaluierung bis zum Studienende beibehalten. Die Therapiezyklen dauerten jeweils 3 Wochen. Zeigte sich dabei ein klinischer Effekt, wurden Dosisexpansionskohorten mit weiteren Patienten gebildet, in denen die Therapie in der betreffenden Dosierung fortgeführt wurde, wenn diese Dosis in akzeptabler Weise verträglich war. Für die Dosisexpansion mussten sich die Patienten nach der ersten Progression einer Tumorbiopsie zur EGFR-T790M-Bestimmung unterzogen haben. Nachdem im März 2013 der erste Patient aufgenommen wurde, war der Datenstichtag für die publizierte Analyse der 1. August 2014.

Die Studie wurde an 33 Zentren in Asien, Australien, USA und Europa durchgeführt. Insgesamt erhielten 253 Patienten (31 in Eskalations-, 222 in Expansionskohorten) , von denen 96\% ein Adenokarzinom aufwiesen und $62 \%$ Frauen waren, $\geq 1$ Dosis AZD9291. Alle Patienten wurden zuvor mit $\geq 1$ TKI und in $80 \%$ der Fälle über Chemotherapie behandelt. $62 \%$ der Patienten der Dosisexpansionskohorten hatten eine T790M-Mutation. Die Gesamtansprechrate betrug $51 \%$. Während bei bestätigten EGFR-T790M-Mutationen 61\% der Patienten auf die Therapie ansprachen, lag das Ansprechen bei fehlender T790MMutation bei $21 \%$.

Die objektiven Ansprechraten unterschieden sich nicht wesentlich zwischen asiatischen und nicht asiatischen Patienten und waren für jede untersuchte Dosierung vergleichbar. EGFR-T790M-positive Patienten hatten ein medianes progressionsfreies Überleben von 9,6 Monaten, Ptienten ohne Mutation erreichten 2,8 Monate. In der Dosisexpansionsphase wurden keine dosislimitierenden Toxizitäten festgestellt. Die häufigsten unerwünschten Nebenwirkungen waren Durchfall (47\%), Hautausschlag (40\%), Übelkeit (22\%) und Appetitverlust (21\%), wobei die Durchfall- und Hautausschlaghäufigkeit dosisabhängig zunahmen. Therapieassoziierte schwere Nebenwirkungen traten bei $6 \%$ der Patienten auf.

\section{Fazit}

Mehr als die Hälfte der Patienten mit fortgeschrittenem NSCLC und der Resistenzmutation T790M, deren Erkrankung unter vorangegangener TKI-Therapie progredierte, sprachen in dieser Studie auf den TKI AZD9291 an. Die noch unreifen Daten zum progressionsfreien Überleben halten die Autoren für vielversprechend.

\section{Was kostet Lungenkrebs?}

Lungenkrebs ist mit jährlich über 50000 Neuerkrankungen eine der häufigsten Krebserkrankungen in Deutschland. Trotzdem liegen bis dato kaum Zahlen zur Versorgungssituation der Betroffenen und den damit verbundenen Kosten vor. Forscher am Helmholtz Zentrum München haben nun anhand von umfangreichen Krankenkassendaten untersucht, was die Erkrankung kostet und welche Behandlung die beste Prognose hat. Die Ergebnisse wurden im September in Lung Cancer publiziert (DOI: 10.1016/j.lungcan.2015.09.005).

Die Wissenschaftler um Dr. L. Schwarzkopf und Prof. R. Leidl analysierten die Daten von über 17000 Lungenkrebspatienten (ca. 12000 Männer und ca. 5000 Frauen). „Erfasst wurden Lungenkrebsfälle aus dem Jahr 2009, deren Entwicklung dann über einen Zeitraum von 3 Jahren beobachtet wurde“, erklärt Schwarzkopf. Dabei registrierten sie Operationen, Chemotherapien und Bestrahlungen. Die Forscher fanden heraus, dass die höchsten Kosten in den ersten 6 Monaten nach der Diagnose anfallen. Dies sei vor allem bedingt durch den stationären Aufenthalt der Betroffenen. Ambulante onkologische Betreuung spiele demnach eine untergeordnete Rolle.

Der durchschnittliche finanzielle Aufwand von Lungenkrebs betrug etwa $20000 €$ pro Fall. Je nach Art der Behandlung variiert dieser Wert aber sehr stark. Insgesamt stellten die Wissenschaftler fest, dass etwa ein Drittel der Patienten durch eine Operation behandelt wurde. Die Prognose dieser Gruppe war im Vergleich mit anderen Behandlungsarten wie Bestrahlung oder Chemotherapie deutlich besser. Die Experten geben allerdings zu bedenken, dass nicht in jedem Fall ein operativer Eingriff möglich oder sinnvoll ist. In diesem Zusammenhang gewinnt nach Ansicht des Forscherteams die Weiterentwicklung von Früherkennungsmaßnahmen an Bedeutung. Denn auf diese Weise erhöht sich die Chance einer Diagnosestellung in einem noch operablen Krankheitsstadium.

Nach einer Mitteilung des Helmhotz Zentrums München 